\title{
EFeito da Umidade do Solo sobre a Capacidade de Canavalia ensiformis E Stizolobium aterrimum EM REMEDIAR SOLOS ConTAMinados COM HeRBICIDAs ${ }^{1}$
}

\author{
Effect of Soil Humidity on Canavalia ensiformis and Stizolobium aterrimum Capacity of \\ Remediating Soils Contaminated by Herbicides
}

\author{
BELO, A.F. ${ }^{2}$, SANTOS, E.A. ${ }^{3}$, SANTOS, J.B. ${ }^{4}$, FERREIRA, L.R. ${ }^{5}$, SILVA, A.A. ${ }^{5}$, CECON, P.R. ${ }^{6}$ \\ e SILVA, L.L. ${ }^{7}$
}

\begin{abstract}
RESUMO - Objetivou-se, neste trabalho, avaliar os efeitos da umidade do solo sobre a capacidade de Canavalia ensiformis e Stizolobium aterrimum em remediar solos contaminados com os herbicidas tebuthiuron e trifloxysulfuron-sodium. O trabalho foi realizado em duas etapas, sendo na primeira avaliado o crescimento de C. ensiformis e $S$. aterrimum em solo com diferentes niveis de umidade, contaminados ou não com herbicidas. Os tratamentos foram compostos pela combinação entre os herbicidas tebuthiuron, trifloxysulfuron-sodium e ausência de herbicida, associados a quatro teores de água do solo $(0,287,0,358,0,431$ e $0,575 \mathrm{~kg} \mathrm{~kg}^{-1}$ ), dispostos em esquema fatorial $3 \times 4$, sob delineamento inteiramente casualizado, com três repetições, para cada espécie remediadora. Após o preparo do substrato e enchimento dos vasos, aplicou-se à superficie do solo o herbicida trifloxysulfuron-sodium ou tebuthiuron. Um dia após essa aplicação, procedeu-se à semeadura das espécies remediadoras. Nessa mesma época, utilizaram-se, como testemunha, vasos sem planta remediadora, porém com os mesmos niveis de umidade e com aplicação do herbicida mantido nas mesmas condições daqueles com plantas remediadoras, as quais foram colhidas 60 dias após semeadura. Nessa ocasião, foram avaliadas a altura e a massa seca da parte aérea (MSPA) dessas espécies. Todo o material colhido foi triturado e incorporado ao solo dos seus respectivos vasos. Na segunda etapa, avaliou-se a capacidade remediadora de C. ensiformis e S. aterrimum. Os tratamentos foram compostos pela combinação entre quatro niveis de umidade e cinco tipos de cultivo prévio: cultivo de $C$. ensiformis e $S$. aterrimum na presença e ausência do herbicida e um tratamento sem cultivo prévio e com aplicação do herbicida, dispostos em esquema fatorial 4 × 5 , sob delineamen to inteiramente casualizado, com três repetições, para cada herbicida avaliado. Amostras de 0,5 kg de solo foram retiradas dos vasos (6 L) utilizados na primeira etapa e colocadas em vasos de 0,5 L. Em seguida, cultivaram-se as espécies indicadoras de resíduo dos herbicidas no solo: sorgo (Sorghum bicolor) para o trifloxysulfuron-sodium e soja (Glycine max) para o tebuthiuron. Estas plantas foram colhidas 20 dias após a semeadura, época em que se avaliaram a altura, a MSPA e o grau de intoxicação delas pelos herbicidas. Canavalia ensiformis não sobreviveu até a época de avaliação (60 DAS) em solo contaminado pelo tebuthiuron, independentemente do nível de umidade mantido no solo. S. aterrimum sobreviveu quando cultivada em solo com teor de água entre 0,287 e 0,358 $\mathrm{kg} \mathrm{kg}^{-1}$ e, quando comparada a $C$. ensiformis, foi mais eficiente na descontaminação do solo com residual do tebuthiuron. Nos solos contaminados com trifloxysulfuron-sodium ou com tebuthiuron, com o cultivo prévio das espécies remediadoras, o crescimento do sorgo e da soja foi melhor se comparado ao daquelas plantas cultivadas no solo onde não foi feito o cultivo das espécies remediadoras. De maneira geral, a variação da umidade não interferiu no processo de remediação, sendo os efeitos observados no desenvolvimento das espécies remediadoras. O melhor desenvolvimento de C. ensiformis e
\end{abstract}

Recebido para publicação em 25.10.2006 e na forma revisada em 15.5.2007.

Eng $^{\mathrm{a}}$-Agra ${ }^{\mathrm{a}}$, Doutoran da em Fitotecnia, Dep. de Fitotecnia da Universidade Federal de Viçosa - DFT/UFV, 36570-000, ViçosaMG,<ferreiragro@yahoo.com.br $>;{ }^{3}$ Eng $^{\mathrm{o}}$-Agro, Mestrando em Fitotecnia - DFT/UFV; ${ }^{4}$ D.S., Prof. da Universidade Vale do Rio Doce - UNIVALE; ${ }^{5}$ D.S., Prof. Associado - DFT/UFV; ${ }^{6}$ D.S., Prof. Adjunto, Dep. de Informática - DPI/UFV; ${ }^{7}$ EngenheiroAgrônomo. 
S. aterrimum foi observado em solo com teor de água mantido em torno de $0,431 \mathrm{~kg} \mathrm{~kg}^{-1}$; contudo, nesse teor de água, o tebuthiuron é mais facilmente disponibilizado para a solução do solo.

Palavras-chave: degradação, persistência no solo, teor de umidade, tebuthiuron, trifloxysulfuron-sodium.

\begin{abstract}
This paper aimed to evaluate the effect of soil humidity on Canavalia ensiformis and Stizolobium aterrimum capacity of remediating soil contaminated with tebuthiuron and trifloxysulfuron-sodium. The experiment was divided into two stages. In the first stage, soil at different levels of humiditywere used, contaminated or not with herbicide, to evaluateC. ensiformis and S. aterrimum growth. Treatments consisted of a combination of tebuthiuron, trifloxysulfuronsodium and herbicide absence, associated to four levels of soil water content $(0.287,0.358,0.431$ and $0.575 \mathrm{~kg} \mathrm{~kg}^{-1}$ ), arranged in a $3 \times 4$ factorial scheme, in a completely randomized design, with three replicates for each species. Trifloxysulfuron-sodium or tebuthiuron were applied on soil surface after preparing the substrate and filling the pots, and the species were sowed one day after that. At this stage, pots with same humidity level and under the same conditions, but without remediating plants, were used as control. After 60 days, plants were harvested to evaluate height and dry matter of ground tissue. All material was ground and incorporated into soil of their respective pots. At the second stage, the remediating capacity of $\mathbf{C}$. ensiformis and $\mathbf{S}$. aterrimum was evaluated. Treatments consisted of five humidity levels and five types of prior cultivation, arranged in a $4 \times 5$ factorial scheme, in a completely randomized design, with three replicates.Samples of $0.5 \mathrm{~kg}$ of soil were taken out from the pots previously used and placed in $0.5 \mathrm{~L}$ pots, where sorghum (Sorghum bicolor) was cultivated to trifloxysulfuron-sodium and soybean (Glycine max) to tebuthiuron. After 20 sowing days, plants were harvested to evaluate height, ground tissue dry matter and their intoxication level. C. ensiformis did not survive up to 60 days after sowing in tebuthiuron-contaminated soil, at any humidity level, and S. aterrimum survived when cultivated in soil between 0.287 and $0.358 \mathrm{~kg} \mathrm{~kg} \mathrm{kumidity}^{-1}$. S. aterrimum was more efficient than $\mathbf{C}$. ensiformis in soil decontamination with tebuthiuron. Sorghum and soybean grew better in soil contaminated with trifloxysulfuron-sodium or tebuthiuron, with prior cultivation, than in soil without prior cultivation. In general, remediation was not affected by humidity variation, with the effects on the development of remediating species being observed. C. ensiformis and S. aterrimum developed best in soil with humidity around $0.431 \mathrm{~kg} \mathrm{~kg}^{-1}$; however, at this level, tebuthiuron is more easily available for the soil solution.
\end{abstract}

Keywords: degradation, humidity content, soil persistence, tebuthiuron, trifloxysulfuron-sodium.

\section{INTRODUÇÃO}

A utilização de plantas como agentes descontaminantes de poluentes orgânicos, como os herbicidas, em solos e recursos hídricos, denominada fitorremediação, desponta como uma técnica promissora, tanto econômica como ambientalmente correta, sendo direcionada até mesmo para a agricultura. Essa técnica apresenta-se como uma alternativa agronômica para sistemas de cultivo que necessitam reduzir a persistência no solo de herbicidas com longo período residual, como o trifloxysulfuron-sodium e o tebuthiuron, que podem persistir no solo por até 8 e 24 meses, respectivamente (Rodrigues \& Almeida, 2005).
Quando os herbicidas são aplicados nos agroecossistemas, eles sofrem uma série de reações e redistribuem-se nos diversos componentes desse ambiente, contaminando-os. Assim, considerando-se os herbicidas aplicados nos solos, o destino deles é governado por três processos gerais (Blanco, 1979): processos físicos: volatilização, lixiviação pela água e erosão com o solo por vento e água; processos químicos: fotodecomposição, adsorção, reações químicas com os constituintes do solo e retirada pelas plantas e microrganismos; e processos microbiológicos, representados pela decomposição microbiana.

Adicionalmente, o manejo do solo também afeta a atividade dos herbicidas residuais e 
influencia a eficácia de controle das plantas daninhas, a persistência e o risco de contaminação ambiental (Levanon et al., 1993; Weed et al., 1995). A atividade dos herbicidas residuais pode variar em razão do tipo e conteúdo de argila, do teor de matéria orgânica e da umidade do solo (Walker et al., 1992). Para alguns herbicidas, o processo de adsorção é dependente do teor de óxidos e hidróxidos de ferro e aluminio principalmente se as cargas de superficie são dependentes do $\mathrm{pH}$. Isso ocorre porque esses fatores afetam a sorção, a lixiviação e a degradação biológica, as quais regulam a concentração, a mobilidade e a persistência de herbicidas no solo (Walker et al., 1992; Mueller et al., 1999). A intensidade com que o manejo afeta a atividade dos herbicidas residuais depende dos efeitos sobre o conteúdo e a infiltração de água, assim como sobre o teor da matéria orgânica do solo. A infiltração de água promove a lixiviação, enquanto a matéria orgânica favorece a sorção dos herbicidas (Walker et al., 1992; Flury, 1996).

A lixiviação constitui um dos principais processos de dissipação dos herbicidas no solo, exercendo influência direta sobre o controle das plantas daninhas, a persistência e o risco de contaminação ambiental. Em adição, propriedades intrínsecas dos herbicidas, dos solos e do clima influenciam a lixiviação, sendo mais suscetiveis os herbicidas com elevada solubilidade em água e fraca adsorção ao solo. O herbicida, para ser lixiviado, deve estar na solução do solo ou adsorvido a pequenas partículas, como argilas, ácidos fúlvicos e húmicos de baixo peso molecular, aminoácidos, peptídeos, açúcares, entre outros (Oliveira, 2001).

Herbicidas solúveis em água, como tebuthiuron e trifloxysulfuron-sodium, tornamse mais disponiveis na solução do solo, facilitando sua absorção pelas plantas; apesar de controlarem as plantas daninhas, eles podem provocar toxicidade em culturas sucessivas. Dessa forma, a utilização de plantas fitorremediadoras em ambientes com resíduo desses herbicidas é de grande importância, por favorecer a descontaminação do solo e permitir a antecipação do plantio de culturas sensiveis.

Objetivou-se com este trabalho avaliar o efeito da umidade do solo sobre a capacidade de $C$. ensiformis e $S$. aterrimum em remediar solos contaminados com os herbicidas tebuthiuron ou trifloxysulfuron-sodium.

\section{MATERIAL E MÉTODOS}

Este trabalho, desenvolvido em casa de vegetação, foi conduzido em duas etapas. Na primeira, visando avaliar o crescimento das espécies remediadoras em solo com diferentes niveis de umidade, após enchimento dos vasos com solo devidamente adubado, em umidade ajustada de acordo com os níveis desejados: $0,287,0,358,0,431$ e 0,575 kg de água por kgde solo, e tratado com trifloxysulfuron-sodium (7,5 $\left.\mathrm{g} \mathrm{ha}^{-1}\right)$, tebuthiuron $\left(1.000,0 \mathrm{~g} \mathrm{ha}^{-1}\right) \mathrm{e} \mathrm{au-}$ sência de herbicida, fez-se a semeadura das es pécies remediadoras (C. ensiformis e $S$. aterrimum). Nessa mesma época, vasos sem planta remediadora, porém com os mesmos niveis de umidade e com aplicação dos herbicidas, foram mantidos nas mesmas condições daqueles com plantas remediadoras. Para avaliação do crescimento das plantas, cada conjunto de vasos com uma espécie remediadora foi considerado um experimento disposto em esquema fatorial $3 \times 4$ (três doses de herbicida e quatro niveis de umidade), sob delineamento inteiramente casualizado, com três repetições. Os diferentes niveis de umidade foram estabelecidos previamente a partir da curva de retenção de água do solo (Figura 1). Utilizaram-se vasos com capacidade para $6 \mathrm{~L}$, preenchidos com 4 kg de solo, cuja caracterização química e fisica é apresentada na Tabela 1.

A semeadura das espécies remediadoras foi realizada um dia após a aplicação do herbicida. Após a emergência delas, foi feito o desbaste, deixando-se três plantas por vaso. Aos 60 dias após a semeadura (DAS), as plantas foram cortadas rente ao solo, sendo avaliadas a altura e a massa seca da parte aérea (MSPA) após secagem das plantas em estufa $\left(70{ }^{\circ} \mathrm{C}\right.$ por $72 \mathrm{~h}$ ). Durante o período de condução do experimento, manteve-se a umidade do solo nos níveis desejados, por meio de pesagens diárias dos vasos com reposição da água consumida. A parte aérea das plantas remediadoras, após secagem, avaliação e moagem, foi devolvida ao respectivo vaso, em razão da possibilidade da presença de resíduo do herbicida nessa região. 


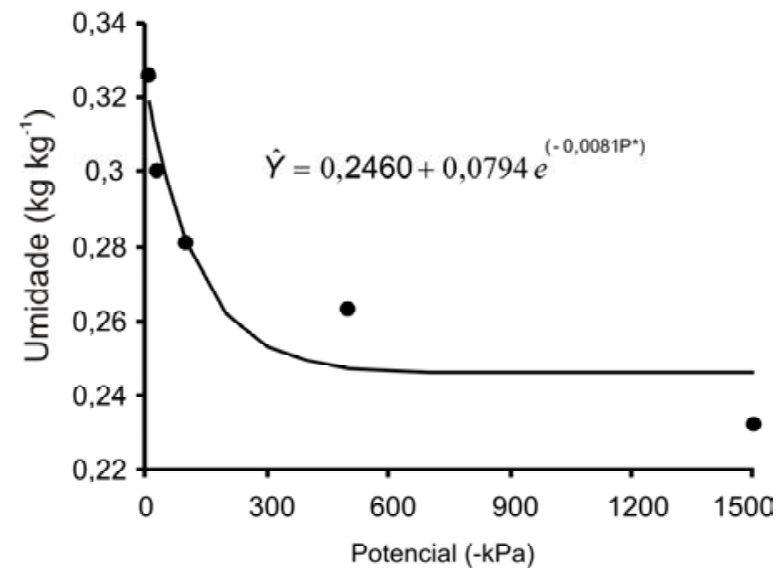

Figura 1 - Curva de retenção de água no Argissolo VermelhoAmarelo utilizado no experimento. $\mathrm{P}^{*}=$ potencial de retenção. e feijão-de-porco (C. ensiformis) na presença e ausência do herbicida e um tratamento sem cultivo prévio e com aplicação do herbicida, dispostos em esquema fatorial 4 x 5 , sob delineamento inteiramente casualizado, com três repetições, sendo um experimento para cada herbicida avaliado. Amostras de $0,5 \mathrm{~kg}$ de solo foram retiradas dos vasos $(6 \mathrm{~L})$ utilizados na primeira etapa e colocadas em vasos de 0,5 L. Em seguida, cultivou-se a espécie indicadora da presença de resíduo de cada herbicida: sorgo (Sorghum bicolor), para bioindicação do trifloxysulfuron-sodium, e soja (Glycine max), para o tebuthiuron. Para desenvolvimento dessas plantas, em todos os tratamentos a umidade do solo foi mantida em torno de $80 \%$ de sua capacidade de campo $\left(0,431 \mathrm{~kg} \mathrm{~kg}^{-1}\right)$. Aos 20 DAS, fez-se a avaliação do grau de intoxicação das

Tabela 1 - Composição físico-química de amostra do solo Argissolo Vermelho-Amarelo utilizado no experimento

\begin{tabular}{|c|c|c|c|c|c|c|c|c|c|c|}
\hline \multicolumn{11}{|c|}{ Análise granulométrica $\left(\right.$ dag $\left.\mathrm{kg}^{-1}\right)$} \\
\hline \multicolumn{2}{|l|}{ Argila } & \multicolumn{2}{|l|}{ Silte } & Areia fina & \multicolumn{3}{|c|}{ Areia grossa } & \multicolumn{3}{|c|}{ Classifica ção textural } \\
\hline \multicolumn{2}{|l|}{39} & 11 & \multicolumn{2}{|c|}{17} & \multicolumn{3}{|c|}{33} & \multicolumn{3}{|c|}{ Argilo-aren osa } \\
\hline \multicolumn{11}{|c|}{ Análi se Química } \\
\hline $\mathrm{pH}$ & $\mathrm{P}$ & $\mathrm{K}^{+}$ & $\mathrm{H}+\mathrm{Al}$ & $\mathrm{Al}^{3+}$ & $\mathrm{Ca}^{2+}$ & $\mathrm{Mg}^{2+}$ & $\mathrm{CTC}_{\text {total }}$ & $\mathrm{V}$ & $\mathrm{m}$ & $\mathrm{MO}$ \\
\hline ----- $\mathrm{H}_{2} \mathrm{O}$----- & \multicolumn{2}{|c|}{$--\mathrm{mg} \mathrm{dm}^{-3}-$} & \multicolumn{5}{|c|}{ - $\mathrm{cmol}_{\mathrm{c}} \mathrm{dm}^{-3}$} & \multicolumn{2}{|c|}{------ \% ------ } & -- dag $\mathrm{kg}^{-1}$-- \\
\hline 5,6 & 157 & 88 & 5,94 & 0,1 & 7,03 & 1,23 & 14,02 & 58,90 & 1,19 & 2,18 \\
\hline
\end{tabular}

Análises realizadas nos Laboratórios de Análises Físicas e Químicas de Solo do Departamento de Solos da UFV, segundo a metodologia descrita pela Empresa Brasileira de Pesquisa Agropecuária-EMBRAPA (1997).

Os dados foram submetidos à análise de variância. A comparação de médias entre as doses dos herbicidas, dentro de cada nível de umidade no solo e para cada espécie, foi realizada por meio do teste de Tukey a $5 \%$ de probabilidade, e o efeito de cada nivel de umidade no solo para cada dose dos herbicidas, por regressão. Os modelos foram escolhidos com base na significância dos coeficientes de regressão, utilizando-se o teste $\mathrm{t}$ a $5 \%$, no fenômeno biológico e no coeficiente de determinação $\left(\mathrm{r}^{2}\right)$.

$\mathrm{Na}$ segunda etapa, avaliou-se a capacidade remediadora de $C$. ensiformis e $S$. aterrimum, verificando o nivel de descontaminação a partir da primeira etapa. Os tratamentos avaliados foram compostos pela combinação entre quatro niveis de umidade e cinco tipos de cultivo prévio: cultivo de mucuna-preta (S. aterrimum) plantas indicadoras pelos herbicidas (atribuindo-se notas variando de zero, para ausência de intoxicação, a 100, para morte da planta). Nessa ocasião, determinou-se ainda a altura e a massa seca da parte aérea das plantas.

Os dados referentes às espécies indicadoras (sorgo e soja) foram submetidos à análise de variância. A comparação de médias entre os tipos de cultivo prévio, dentro de cada nivel de umidade no solo, foi realizada por meio do teste de Tukey a $5 \%$, e o efeito de cada nível de umidade do solo para cada tipo de cultivo prévio, por regressão. Os modelos foram escolhidos com base na significância dos coeficientes de regressão, utilizando-se o teste t a 5\%, no fenômeno biológico e no coeficiente de determinação $\left(\mathrm{r}^{2}\right)$. 


\section{RESULTADOS E DISCUSSÃO}

Verificou-se efeito significativo da umidade no solo contaminado por trifloxysulfuron-sodium ou tebuthiuron sobre a altura e MSPA da espécie remediadora C. ensiformis (Tabela 2). Na presença do trifloxysulfuron-sodium, essa espécie teve a altura e MSPA reduzidas quando comparada ao tratamento sem herbicida. Nos tratamentos que receberam o tebuthiuron, independentemente do nivel de umidade no solo, C. ensiformis não se desenvolveu até a época de avaliação (60 DAS - Tabela 2). não permitindo o crescimento de S. aterrimum até a época de avaliação (60 DAS). Entretanto, pode-se inferir que essa espécie apresenta maior tolerância a esse produto, comparada a C. ensiformis, a qual não se desenvolveu até a época de avaliação, independentemente do nível de umidade.

Quando se avaliou o comportamento das espécies remediadoras com o aumento da umidade (Figura 2), observou-se que em solo com presença e ausência do herbicida trifloxysulfuron-sodium o aumento de umidade interferiu

Tabela 2 - Valores médios de altura e massa seca da parte aérea de plantas de Canavalia ensiformis cultivadas em solo mantido com diferentes níveis de umidade, com e sem aplicação de trifloxysulfuron -sodium ou tebuthiuron, com os respectivos coeficientes de variação

\begin{tabular}{|c|c|c|c|c|c|c|}
\hline \multirow{2}{*}{$\begin{array}{c}\text { Umida de no } \\
\text { solo } \\
\left(\mathrm{kg} \mathrm{kg}^{-1}\right)\end{array}$} & \multicolumn{3}{|c|}{ Altura das plantas $(\mathrm{cm})$} & \multicolumn{3}{|c|}{ Massa seca da parte aérea $(\mathrm{g})$} \\
\hline & $\begin{array}{c}\text { Trifl oxysul furon - } \\
\text { sodium }\end{array}$ & Tebuthiuron & $\begin{array}{c}\text { Sem } \\
\text { herbicida }\end{array}$ & $\begin{array}{l}\text { Trifl oxysul furon - } \\
\text { sodium }\end{array}$ & Tebuthiuron & $\begin{array}{c}\text { Sem } \\
\text { herbicida }\end{array}$ \\
\hline 0,287 & $55,00 \mathrm{~b}$ & $0,00 \mathrm{c}$ & $128,33 \mathrm{a}$ & $9,72 \mathrm{~b}$ & $0,00 \mathrm{c}$ & $24,80 \mathrm{a}$ \\
\hline 0,358 & $106,67 \mathrm{~b}$ & $0,00 \mathrm{c}$ & $188,33 \mathrm{a}$ & $15,85 \mathrm{~b}$ & $0,00 \mathrm{c}$ & $32,37 \mathrm{a}$ \\
\hline 0,431 & $101,67 \mathrm{~b}$ & $0,00 \mathrm{c}$ & $203,33 \mathrm{a}$ & $20,19 \mathrm{~b}$ & $0,00 \mathrm{c}$ & 43,72 a \\
\hline 0,575 & $76,33 \mathrm{~b}$ & $0,00 \mathrm{c}$ & $188,33 \mathrm{a}$ & $14,67 \mathrm{~b}$ & $0,00 \mathrm{c}$ & $41,04 \mathrm{a}$ \\
\hline CV $(\%)$ & \multicolumn{3}{|c|}{9,24} & \multicolumn{3}{|c|}{19,70} \\
\hline
\end{tabular}

Médias seguidas pela mesma letra na linha, para cada variável, não diferem entre si pelo teste de Tukey a 5\%.

Em todos os níveis de umidade testados, os valores para altura e MSPA de S. aterrimum na presença do trifloxysulfuron-sodium foram semelhantes aos observados no tratamento sem herbicida, mostrando que não houve efeito desse herbicida no seu crescimento (Tabela 3). Na presença do tebuthiuron, observou-se crescimento de $S$. aterrimum nos menores teores de água $\left(0,287\right.$ e $\left.0,358 \mathrm{~kg} \mathrm{~kg}^{-1}\right)$, porém pouco expressivo em relação ao observado no solo sem herbicida. Enquanto nos vasos contendo solo sem herbicida a altura e MSPA observadas para o teor de $0,287 \mathrm{~kg} \mathrm{~kg}^{-1}$ de água no solo foram, respectivamente, de 165,00 cm e 12,44 g, na presença do tebuthiuron esses valores foram de 39,67 cm e 3,02 g, ou seja, em torno de $25 \%$ dos valores observados no tratamento controle (Tabela 3). Segundo Rodrigues \& Almeida (2005), o tebuthiuron apresenta elevada solubilidade em água $\left(2.570 \mathrm{mg} \mathrm{L}^{-1}\right.$ a $\left.20^{\circ} \mathrm{C}\right)$, podendo, neste trabalho, ter influenciado sua disponibilidade nas maiores umidades testadas, no crescimento de C. ensiformis. Na presença de trifloxysulfuron-sodium, estimou-se que a altura máxima foi de $110,55 \mathrm{~cm}$ no teor de água de $0,444 \mathrm{~kg} \mathrm{~kg}^{-1} \mathrm{e}$, na ausência deste, de $212,50 \mathrm{~cm}^{\mathrm{com}} 0,472 \mathrm{~kg} \mathrm{~kg}^{-1}$ (Figura 2). Na presença desse herbicida, o maior valor de MSPA obtido $(19,97 \mathrm{~g})$ foi no teor de água de $0,455 \mathrm{~kg} \mathrm{~kg}^{-1} \mathrm{e}$, na ausência $(42,74 \mathrm{~g})$, com $0,449 \mathrm{~kg} \mathrm{~kg}^{-1}$ (Figura 3).

Com o aumento da umidade do solo, observou-se acréscimo na MSPA de S. aterrimum na presença e ausência do herbicida trifloxysulfuron-sodium. Na presença desse herbicida, foi estimado que o maior valor de MSPA $(28,71 \mathrm{~g})$ foi obtido quando a espécie foi cultivada em solo com teor de água de $0,494 \mathrm{~kg} \mathrm{~kg}^{-1}$. Na ausência de herbicida, a maior produção de MSPA $(28,25 \mathrm{~g})$ foi obtida quando cultivada em solocom teor de água de $0,468 \mathrm{~kg} \mathrm{~kg}^{-1}$. Nos teores de água superiores a esses observou-se tendência em reduzir a produção de MSPA (Figura 4). 
Tabela 3 - Valores médios de altura e massa seca da parte aérea de plantas de Stizolobium aterrimum cultivadas em solo mantido com diferentes níveis de umidade, com e sem aplicação de trifloxysulfuron-sodium ou tebuthiuron, com os respectivos coeficientes de variação

\begin{tabular}{|c|c|c|c|c|c|c|}
\hline \multirow{2}{*}{$\begin{array}{l}\text { Umidade } \\
\text { no solo } \\
\left(\mathrm{kg} \mathrm{kg}^{-1}\right)\end{array}$} & \multicolumn{3}{|c|}{ Altura das plantas (cm) } & \multicolumn{3}{|c|}{ Massa seca da parte aérea $(\mathrm{g})$} \\
\hline & $\begin{array}{l}\text { Trifl oxysulfuron - } \\
\text { sodium }\end{array}$ & Tebuthiuron & $\begin{array}{c}\text { Sem } \\
\text { herbicida }\end{array}$ & $\begin{array}{l}\text { Trifl oxysul furon - } \\
\text { sodium }\end{array}$ & Tebuthiuron & $\begin{array}{c}\text { Sem } \\
\text { herbicida }\end{array}$ \\
\hline 0,287 & $178,33 \mathrm{a}$ & $39,67 \mathrm{~b}$ & $165,00 \mathrm{a}$ & $12,98 \mathrm{a}$ & $3,02 \mathrm{~b}$ & $12,44 \mathrm{a}$ \\
\hline 0,358 & $183,33 \mathrm{a}$ & $24,45 \mathrm{~b}$ & $176,67 \mathrm{a}$ & $23,78 \mathrm{a}$ & $2,46 \mathrm{~b}$ & 24,32 a \\
\hline 0,431 & $195,68 \mathrm{a}$ & $0,00 \mathrm{~b}$ & 194,99 a & $26,15 \mathrm{a}$ & $0,00 \mathrm{~b}$ & $26,41 \mathrm{a}$ \\
\hline 0,575 & $191,67 \mathrm{a}$ & $0,00 \mathrm{~b}$ & $181,67 \mathrm{a}$ & $26,57 \mathrm{a}$ & $0,00 \mathrm{~b}$ & $23,09 \mathrm{a}$ \\
\hline $\mathrm{CV}(\%)$ & \multicolumn{3}{|c|}{8,55} & \multicolumn{3}{|c|}{9,65} \\
\hline
\end{tabular}

Médias seguidas pela mesma letra na linha, para cada variável, não di ferem entre si pelo teste de Tukey a $5 \%$.

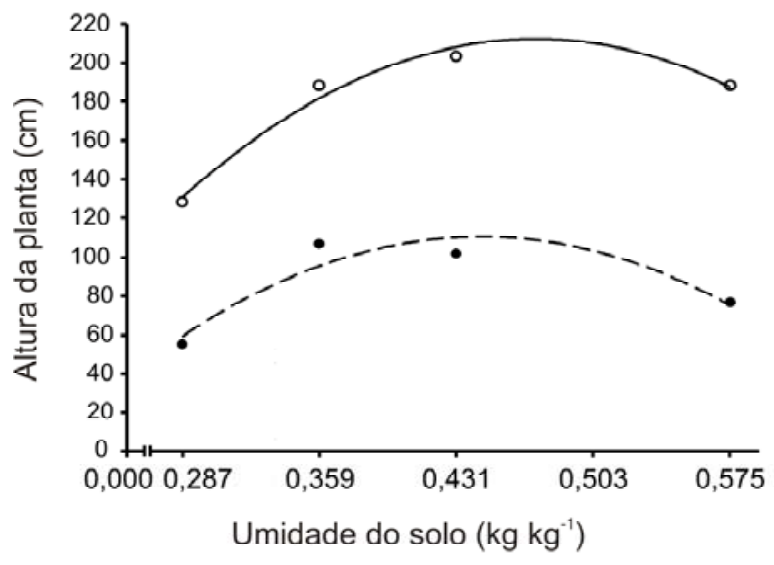

Figura 2 - Valor estimado e observado para altura de plantas de Canavalia ensiformis cultivadas em solo com e sem aplicação de trifloxysulfuron-sodium, em função de diferentes níveis de umidade (U). (com herbicida: - $-\hat{\mathrm{Y}}=-298,73+1843,7 \mathrm{U}-2076,3 * \mathrm{U}^{2}, \mathrm{r}^{2}=0,87$; e sem herbicida: $\mathrm{o}-\hat{\mathrm{Y}}=-317,09+2242,4 \mathrm{U}-2373,67^{*} \mathrm{U}^{2}, \mathrm{r}^{2}$ $=0,97)$.

Quanto ao herbicida tebuthiuron, aos 20 dias após a semeadura (DAS) da espécie indicadora observaram-se os efeitos das espécies remediadoras e dos diferentes niveis de umidade sobre a altura, a MSPA e os sintomas de intoxicação nas plantas de soja. Plantas cultivadas nos solos anteriormente remediados pelas espécies $C$. ensiformis e $S$. aterrimum evidenciaram menor disponibilidade desse herbicida na solução do solo, permitindo melhor crescimento da soja em comparação ao tratamento nãofitorremediado. Nessas condições, verificou-se que a altura e a MSPA das plantas de soja foram maiores que no solo sem cultivo prévio. No que

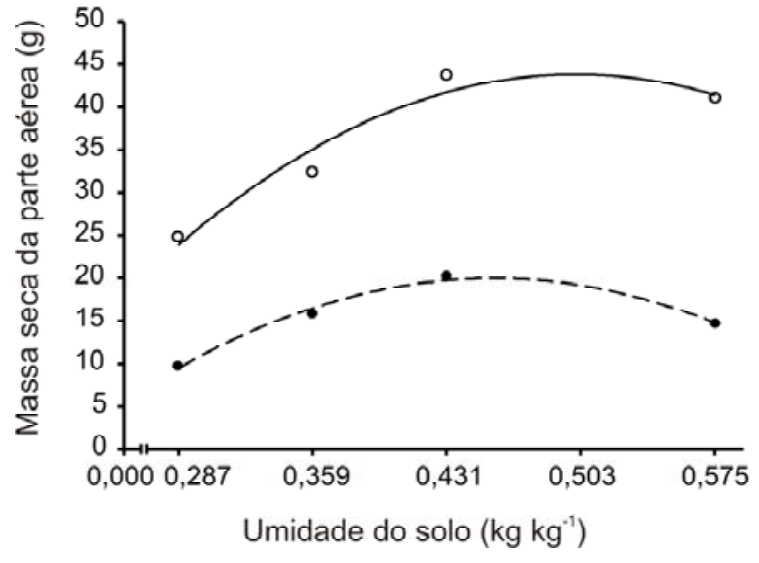

Figura 3 - Valor estimado e observado para massa seca da parte aérea de plantas de Canavalia ensiformis cultivadas em solo com e sem aplicação de trifloxysul furon-s odium, em função de diferentes níveis de umidade (U). (com herbicida: $\bullet-\hat{Y}=-56,432+$ $335,24 * U-367,74 * U^{2}, r^{2}=0,99$; e sem herbicida: o $\left.\hat{\mathrm{Y}}=-66,919+443,47 * \mathrm{U}-443,73 * \mathrm{U}^{2}, \mathrm{r}^{2}=0,95\right)$.

se refere ao efeito do teor de água no solo nos tratamentos anteriormente remediados por S. aterrimum, as plantas de soja apresentaram maior crescimento quando cultivadas em solo com maior teor de água (Tabela 4). Admitindo que o tebuthiuron apresente maior taxa de degradação em solos mais úmidos (Rodrigues \& Almeida, 2005), esperava-se mesmo que houvesse, no maior teor de água no solo, menor residual desse produto.

Avaliando a intoxicação das plantas de soja, verificou-se que $S$. aterrimum foi mais eficiente na descontaminação do solo, quando comparada 


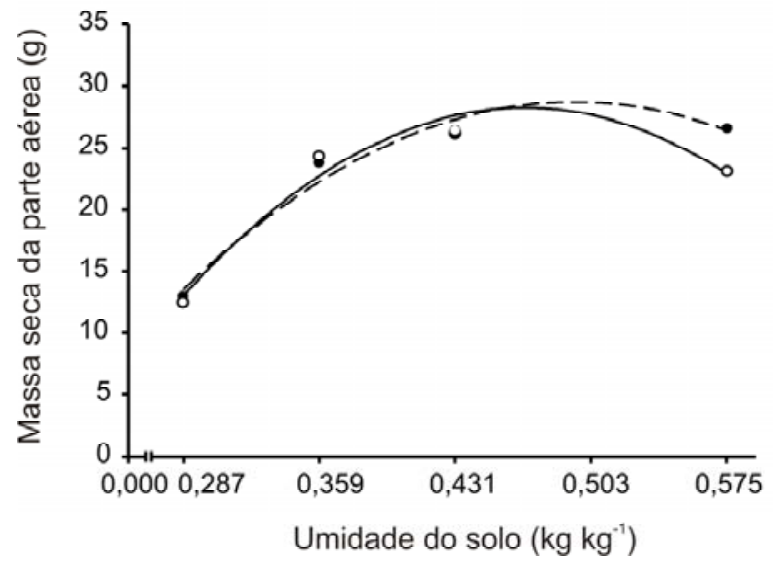

Figura 4 - Valor estimado e observado para massa seca da parte aérea de plantas de Stizolobium aterrimum cultivadas em solo com e sem aplicação de trifloxysulfuron-sodium, em função de diferentes níveis de umidade $(\mathrm{U})$. (com herbicida: $\bullet-\hat{\mathrm{Y}}=-57,636+$ $349,86 \mathrm{U}-354,36 * \mathrm{U}^{2}, \mathrm{r}^{2}=0,94$; e sem herbicida: $\mathrm{o}-\hat{\mathrm{Y}}=$ $\left.-73,588+435,65 \mathrm{U}-465,87 * \mathrm{U}^{2}, \mathrm{r}^{2}=0,95\right)$. a C. ensiformis. Foi observada maior severidade dos sintomas de intoxicação nas plantas de soja cultivadas em solo remediado por C. ensiformis mantido anteriormente no menor teor de água $\left(0,287 \mathrm{~kg} \mathrm{~kg}^{-1}\right)$. Admitindo-se que, no cultivo da soja, a umidade do solo foi padronizada para $0,431 \mathrm{~kg} \mathrm{~kg}^{-1}$, é provável que o herbicida - que anteriormente não foi disponibilizado totalmente para C. ensiformis, por estar fortemente adsorvido à argila do solo - tenha se tornado disponível para a soja devido à maior disponibilidade de água, o que promoveu a liberação do herbicida para solução do solo (Tabela 4). Rocha (2003), estudando a sorção de 2,4-D e diuron, verificou que a mineralização desses herbicidas foi maior nas amostras com maior teor de água, sendo o 2,4-D mais mineralizado que o diuron.

Observa-se na Figura 5 que, até o teor de água de $0,441 \mathrm{~kg} \mathrm{~kg}^{-1}$, houve aumento na MSPA de plantas de soja no solo cultivado com

Tabela 4 - Valores médios de massa seca da parte aérea (MSPA), altura e intoxicação de plantas de soja aos 20 dias após semeadura, cultivadas em solo mantido com diferentes níveis de umidade, com e sem aplicação de tebuthiuron, remediados ou não por Canavalia ensiformis e Stizolobium aterrimum por 60 dias após aplicação do herbicida, com os respectivos coeficientes de variação

\begin{tabular}{|c|c|c|c|c|c|c|}
\hline \multirow{2}{*}{$\begin{array}{l}\text { Característica } \\
\text { avaliada }\end{array}$} & \multirow{2}{*}{$\begin{array}{c}\text { Umidade do } \\
\text { solo } \\
\left(\mathrm{kg} \mathrm{kg}^{-1}\right)\end{array}$} & \multicolumn{2}{|c|}{ Canavalia ensiformis } & \multicolumn{2}{|c|}{ Stizolobium aterrimum } & \multirow{2}{*}{$\begin{array}{c}\begin{array}{c}\text { Sem cultivo } \\
\text { prévio }\end{array} \\
\text { Tebuthiuron }\end{array}$} \\
\hline & & Tebuthiuron & Sem herbicida & Tebuthiuron & Sem herbicida & \\
\hline \multirow{4}{*}{$\operatorname{MSPA}(\mathrm{g})$} & 0,287 & $0,307 \mathrm{c}$ & $0,463 \mathrm{a} \mathrm{b}$ & $0,383 \mathrm{~b} \mathrm{c}$ & 0,497 a & $0,253 \mathrm{~d}$ \\
\hline & 0,358 & $0,360 \mathrm{~b}$ & $0,567 \mathrm{a}$ & $0,333 \mathrm{~b}$ & $0,627 \mathrm{a}$ & $0,031 \mathrm{c}$ \\
\hline & 0,431 & $0,317 \mathrm{c}$ & $0,450 \mathrm{~b}$ & $0,320 \mathrm{c}$ & $0,660 \mathrm{a}$ & $0,071 \mathrm{~d}$ \\
\hline & 0,575 & $0,327 \mathrm{c}$ & $0,570 \mathrm{a}$ & $0,413 \mathrm{~b}$ & $0,540 \mathrm{a}$ & $0,031 \mathrm{~d}$ \\
\hline \multicolumn{2}{|l|}{ CV $(\%)$} & \multicolumn{5}{|c|}{ - } \\
\hline \multirow{4}{*}{ Altura $(\mathrm{cm})$} & 0,287 & $14,40 \mathrm{a}$ & $16,03 \mathrm{a}$ & $17,23 \mathrm{a}$ & 16,77 a & $4,00 \mathrm{~b}$ \\
\hline & 0,358 & $19,93 \mathrm{a} \mathrm{b}$ & $16,70 \mathrm{~b}$ & $16,77 \mathrm{~b}$ & $21,63 \mathrm{a}$ & $3,00 \mathrm{c}$ \\
\hline & 0,431 & $15,47 \mathrm{~b}$ & $16,13 \mathrm{~b}$ & $16,93 \mathrm{~b}$ & 21,27 a & $5,00 \mathrm{c}$ \\
\hline & 0,575 & $15,37 \mathrm{~b}$ & $18,17 \mathrm{a} \mathrm{b}$ & $17,41 \mathrm{a} \mathrm{b}$ & $18,80 \mathrm{a}$ & $5,17 \mathrm{c}$ \\
\hline \multicolumn{7}{|l|}{$\mathrm{CV}(\%)$} \\
\hline \multirow{4}{*}{ Intoxicação (\%) } & 0,287 & $80,00 \mathrm{~b}$ & $0,00 \mathrm{~d}$ & $26,67 \mathrm{c}$ & $0,00 \mathrm{~d}$ & 92,67 a \\
\hline & 0,358 & $30,00 \mathrm{~b}$ & $0,00 \mathrm{c}$ & $34,00 \mathrm{~b}$ & $0,00 \mathrm{c}$ & 93,33 a \\
\hline & 0,431 & $56,67 \mathrm{~b}$ & $0,00 \mathrm{~d}$ & $48,33 \mathrm{c}$ & $0,00 \mathrm{~d}$ & 86,67 a \\
\hline & 0,575 & $70,00 \mathrm{~b}$ & $0,00 \mathrm{~d}$ & $23,33 \mathrm{c}$ & $0,00 \mathrm{~d}$ & 89,00 a \\
\hline \multicolumn{2}{|l|}{ CV $(\%)$} & \multicolumn{5}{|c|}{------------------------------------------------ 8,88 -------- } \\
\hline
\end{tabular}

Médias seguidas pela mesma letra, em cada linha, não diferem entre si pelo teste de Tukey a 5\%. * Para a bioindicação do herbicida durante o cultivo da soja, a umidade do solo foi padronizada para $0,431 \mathrm{~kg} \mathrm{~kg}^{-1}$. 


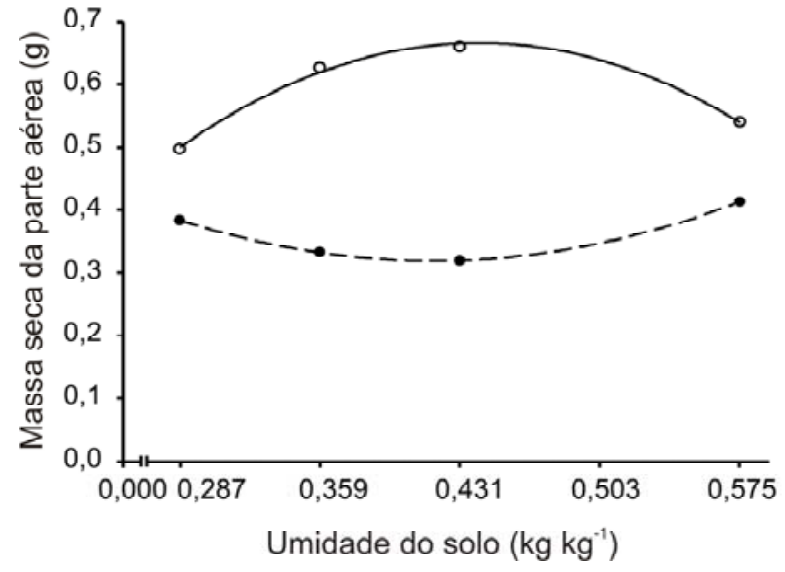

Figura 5 - Valor estimado e observado para massa seca da parte aérea de plantas de soja cultivadas em solo com e sem aplicação de tebuthiuron, em função de diferentes níveis de umidade (U), após 60 dias de remediação do solo pela espécie Stizolobium aterrimum (com herbicida: - $-\hat{\mathrm{Y}}=0,9726-3,1306 \mathrm{U}+3,7519 * \mathrm{U}^{2}, \mathrm{r}^{2}=0,97$; e sem herbicida: $\mathrm{o}-\hat{\mathrm{Y}}=-0,7053+6,2295 \mathrm{U}-7,0706^{*} \mathrm{U}^{2}, \mathrm{r}^{2}=$ $0,96)$.

S. aterrimumque não recebeu tebuthiuron. No solo que foi anteriormente tratado com tebuthiuron e cultivado com S. aterrimum, foi observado que as plantas de soja apresentavam menor valor de MSPA quando essa cultura foi cultivada em solo com teor de água de $0,417 \mathrm{~kg} \mathrm{~kg}^{-1}$. Como S. aterrimum se desenvolveu na época de avaliação (60 DAS) até o teor de água de $0,358 \mathrm{~kg} \mathrm{~kg}^{-1}$, acredita-se que o herbicida não degradado anteriormente por S. aterrimum tenha ficado disponivel para soja.

Na Figura 6, observa-se que o poder remediador de $S$. aterrimum para tebuthiuron resultou em menor índice de intoxicação das plantas de soja, quando comparado ao crescimento desta em solo onde não houve cultivo prévio de espécies remediadoras. Verificouse ainda que o efeito dos niveis de umidade do solo não foi significativo nas variáv eis analisadas para os tipos de cultivo prévio com C. ensiformis na ausência e presença do herbicida tebuthiuron. Os valores médios de MSPA, altura e intoxicação podem ser observados na Tabela 5.

Vinte dias após a semeadura da espécie indicadora em solo sob contaminação do

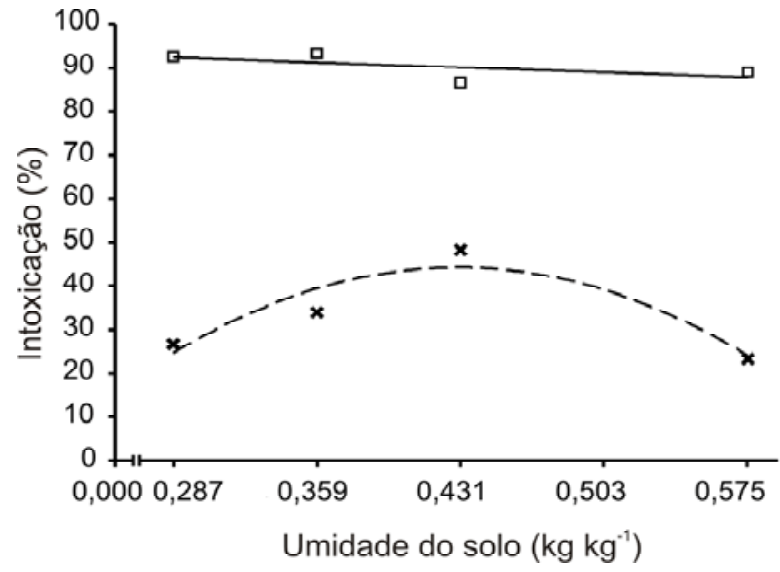

Figura 6 - Valor estimado e observado para intoxicação de plantas de soja cultivadas em solo com aplicação de tebuthiuron, em função de diferentes níveis de umidade (U), após 60 dias de remediação do solo pela espécie Stizolobium aterrimum $(\mathbf{X}-\hat{\mathrm{Y}}=-134,22+830,8 \mathrm{U}-$ $\left.966,32 * U^{2}, r^{2}=0,87\right)$ e sem cultivo prévio de espécies fitorremediad ora $\mathrm{s}\left(\square-\hat{\mathrm{Y}}=94,8333-1,76667^{*} \mathrm{U}, \mathrm{r}^{2}=\right.$ $0,97)$.

herbicida trifloxysulfuron-sodium, constataram-se efeitos das espécies remediadoras e dos diferentes niveis de umidade sobre altura, MSPA e grau de intoxicação das plantas de sorgo utilizadas na bioindicação desse herbicida (Tabela 6).

A fitorremediação do solo contaminado pelo trifloxysulfuron-sodium foi eficiente por ambas as espécies remediadoras, independentemente do nivel de umidade adotado (Tabela 6). Isso ficou evidenciado pelo fato de que plantas de sorgo cultivadas em amostras de solo oriundas de vasos que receberam o trifloxysulfuronsodium, sem cultivo prévio das espécies remediadoras, tiveram seu crescimento severamente reduzido. Tanto a altura quanto a MSPA das plantas de sorgo não variaram onde houve cultivo prévio de C. ensiformis ou $S$. aterrimum, não sendo observados, em ambos os casos, sintomas de intoxicação (Tabela 6). Também Procópio et al. (2005) verificaram que, entre 15 espécies de adubos verdes utilizados em programa de fitorremediação desse herbicida, somente $C$. ensiformis e S. aterrimum possibilitaram o cultivo seqüencial de plantas de feijão, sem prejuízos para biomassa, altura e toxidez da parte aérea, em solo com a umidade em 
Tabela 5 - Equações ajustadas e respectivos coeficientes de determinação para massa seca da parte aérea, altura e intoxicação de plantas de soja aos 20 dias após semeadura em solo com diferentes níveis de umidade (U), com e sem aplicação de tebuthiuron, remediados ou não pelas espécies Canavalia ensiformis e Stizolobium aterrimum por 60 dias após aplicação do herbicida

\begin{tabular}{|l|l|c|}
\hline Tratamento & Equação & $\mathrm{r}^{2}$ \\
\hline & Massa seca da parte aérea (g) & 0,97 \\
\hline Stizolobium aterrimum na presença de tebuthiuron & $\hat{\mathrm{Y}}=0,9726-3,1306 \mathrm{U}+3,751 \mathrm{U}^{2}$ & 0,96 \\
\hline Stizolobium aterrimum na ausência de tebuthiuron & $\hat{\mathrm{Y}}=-0,7053+6,2295 \mathrm{U}-7,0706 \mathrm{U}^{2}$ & - \\
\hline Canavalia ensiformis na presença de tebuthiuron & $\hat{\mathrm{Y}}=0,328$ & - \\
\hline Canavalia ensiformis na ausência de te buthiuron & $\hat{\mathrm{Y}}=0,513$ & - \\
\hline Sem cultivo prévio e com presença de tebuthiuron & $\hat{\mathrm{Y}}=0,040$ & \\
\hline & $\mathbf{A l t u r a} \mathbf{d e}$ plant as (cm) & - \\
\hline Stizolobium aterrimum na presença de tebuthiuron & $\hat{\mathrm{Y}}=17,09$ & - \\
\hline Stizolobium aterrimum na ausência de tebuthiuron & $\hat{\mathrm{Y}}=19,62$ & - \\
\hline Canavalia ensiformis na presença de tebuthiuron & $\hat{\mathrm{Y}}=16,29$ & - \\
\hline Canavalia ensiformis na ausência de tebuthiuron & $\hat{\mathrm{Y}}=16,76$ & - \\
\hline Sem cultivo prévio e com presença de tebuthiuron & $\hat{\mathrm{Y}}=4,29$ & - \\
\hline & Intoxicação (\%) & - \\
\hline Stizolobium aterrimum na presença de tebuthiuron & $\hat{\mathrm{Y}}=-8,41669+40,85 \mathrm{U}-8,08334 \mathrm{U}^{2}$ & \\
\hline Stizolobium aterrimum na ausência de tebuthiuron & $\hat{\mathrm{Y}}=0,00$ & 0,62 \\
\hline Canavalia ensiformis na presença de tebuthiuron & $\hat{\mathrm{Y}}=59,17$ & - \\
\hline Canavalia ensiformis na ausência de tebuthiuron & $\hat{\mathrm{Y}}=0,00$ & - \\
\hline Sem cultivo prévio e com presença de tebuthiuron & $\hat{\mathrm{Y}}=94,8333-1,76667 \mathrm{U}$ & - \\
\hline
\end{tabular}

torno de $80 \%$ da capacidade de campo e contaminado com até duas vezes a dosagem comercialmente recomendada.

O aumento da umidade do solo só foi significativo para altura de plantas de sorgo cultivadas em solos contaminados com o herbicida trifloxysulfuron-sodium, não remediados por C. ensiformis ou S. aterrimum(Tabela 7). Maior valor de altura foi observado para as plantas de sorgo cultivadas em solos mantidos anteriormente na maior umidade. Como não houve cultivo prévio das espécies remediadoras, acredita-se que a maior disponibilidade de água tenha contribuído para ativar a degradação das moléculas do herbicida (Figura 7). Segundo
Rodrigues \& Almeida (2005), o trifloxysulfuronsodium degrada no solo inicialmente por hidrólise química.

Dessa forma, os resultados indicam que, para o tebuthiuron, o teor de água em torno de $0,431 \mathrm{~kg} \mathrm{~kg}^{-1}$ promoveu baixo crescimento nas plantas de soja, provavelmente em razão da maior disponibilidade desse produto na solução do solo. S. aterrimum foi mais eficiente na descontaminação do solo com residual do tebuthiuron, comparada a C. ensiformis. Os teores de água de 0,287, 0,358, 0,431 e $0,575 \mathrm{~kg} \mathrm{~kg}^{-1}$ não interferem na remediação do herbicida trifloxysulfuron-sodium pelas espécies vegetais $C$. ensiformis e $S$. aterrimum. 
Tabela 6 - Valores médios de massa seca da parte aérea (MSPA), altura e intoxicação de plantas de sorgo aos 20 dias após semeadura, cultivadas em solo com diferentes níveis de umidade, com e sem aplicação de trifloxysulfuron -sodium, remediados ou não por Canavalia ensiformis e Stizolobium aterrimum por 60 dias após aplicação do herbicida, com os respectivos coeficientes de variação

\begin{tabular}{|c|c|c|c|c|c|c|}
\hline \multirow{2}{*}{$\begin{array}{l}\text { Característica } \\
\text { avaliada }\end{array}$} & \multirow{2}{*}{$\begin{array}{l}\text { Umidade do } \\
\text { solo* } \\
\left(\mathrm{kg} \mathrm{kg}^{-1}\right)\end{array}$} & \multicolumn{2}{|c|}{ Canavalia ensiformis } & \multicolumn{2}{|c|}{ Stizolobium aterrimum } & \multirow{2}{*}{$\begin{array}{c}\begin{array}{c}\text { Sem cultivo } \\
\text { prévio }\end{array} \\
\begin{array}{c}\text { Trifloxysulfuron- } \\
\text { sodium }\end{array}\end{array}$} \\
\hline & & $\begin{array}{c}\text { Trifloxysulfuron- } \\
\text { sodium }\end{array}$ & $\begin{array}{c}\text { Sem } \\
\text { herbicida }\end{array}$ & $\begin{array}{l}\text { Trifloxysulfuron- } \\
\text { sodium }\end{array}$ & $\begin{array}{c}\text { Sem } \\
\text { herbicida }\end{array}$ & \\
\hline \multirow{4}{*}{ MSPA (g) } & 0,287 & $0,317 \mathrm{ab}$ & $0,267 \mathrm{~b}$ & $0,357 \mathrm{ab}$ & $0,370 \mathrm{a}$ & $0,014 \mathrm{c}$ \\
\hline & 0,358 & $0,360 \mathrm{a}$ & $0,333 \mathrm{a}$ & $0,327 \mathrm{a}$ & $0,297 \mathrm{a}$ & $0,036 \mathrm{~b}$ \\
\hline & 0,431 & $0,317 \mathrm{ab}$ & $0,243 \mathrm{~b}$ & $0,353 \mathrm{ab}$ & $0,253 \mathrm{~b}$ & $0,044 \mathrm{c}$ \\
\hline & 0,575 & $0,388 \mathrm{a}$ & $0,323 \mathrm{a}$ & $0,337 \mathrm{a}$ & $0,313 \mathrm{a}$ & $0,052 \mathrm{~b}$ \\
\hline \multicolumn{2}{|l|}{$\overline{C V}(\%)$} & \multicolumn{5}{|c|}{ 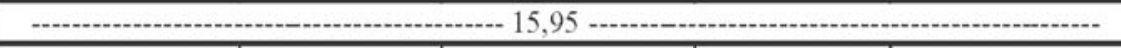 } \\
\hline \multirow{4}{*}{ Altura $(\mathrm{cm})$} & 0,287 & $18,02 \mathrm{a}$ & $18,05 \mathrm{a}$ & $17,97 \mathrm{a}$ & $18,05 \mathrm{a}$ & $5,00 \mathrm{~b}$ \\
\hline & 0,358 & $18,03 \mathrm{a}$ & 18,07 a & $18,03 \mathrm{a}$ & $17,98 \mathrm{a}$ & $5,67 \mathrm{~b}$ \\
\hline & 0,431 & $18,08 \mathrm{a}$ & $18,03 \mathrm{a}$ & $18,08 \mathrm{a}$ & $18,00 \mathrm{a}$ & $9,83 \mathrm{~b}$ \\
\hline & 0,575 & $18,04 \mathrm{a}$ & $18,06 \mathrm{a}$ & $17,92 \mathrm{a}$ & $18,01 \mathrm{a}$ & $10,67 \mathrm{~b}$ \\
\hline \multicolumn{7}{|l|}{$\overline{C V}(\%)$} \\
\hline \multirow{4}{*}{ Intoxicação (\%) } & 0,287 & 0,00 & 0,00 & 0,00 & 0,00 & 85,00 \\
\hline & 0,358 & 0,00 & 0,00 & 0,00 & 0,00 & 92,00 \\
\hline & 0,431 & 0,00 & 0,00 & 0,00 & 0,00 & 90,00 \\
\hline & 0,575 & 0,00 & 0,00 & 0,00 & 0,00 & 85,00 \\
\hline
\end{tabular}

Médias seguidas pela mesma letra, em cada linha, não diferem entre si pelo teste de Tukey a 5\%. * Para a bioindicação do herbi cida durante o cultivo do sorgo, a umidade do solo foi padronizada para $0,431 \mathrm{~kg} \mathrm{~kg}^{-1}$.

Tabela 7 - Equações ajustadas para massa seca da parte aérea, altura e intoxicação de plantas de sorgo aos 20 dias após semeadura, em solo com diferentes níveis de umidade (U), com e sem aplicação de trifloxysulfuron -sodium, remediados ou não pelas espécies Canavalia ensiformis e Stizolobium aterrimum por 60 dias após aplicação do herbicida

\begin{tabular}{|c|c|c|}
\hline Tratamento & Equação & $\mathrm{r}^{2}$ \\
\hline & Massa seca da parte aérea (g) & \\
\hline Stizolobium aterrimum na presença de trifloxysulfuron-sodium & $\hat{\mathrm{Y}}=0,343$ & - \\
\hline Stizolobium aterrimum na ausência de trifloxysulfuron-sodium & $\hat{\mathrm{Y}}=0,308$ & - \\
\hline Canavalia ensiformis na presença de trifloxysulfuron-sodium & $\hat{\mathrm{Y}}=0,346$ & - \\
\hline Canavalia ensiformis na ausência de trifloxysul furon-sodium & $\hat{\mathrm{Y}}=0,292$ & - \\
\hline \multirow[t]{2}{*}{ Sem cultivo prévio e com presença de trifloxysulfuron-sodium } & $\hat{\mathrm{Y}}=0,036$ & - \\
\hline & Altura de plantas (cm) & \\
\hline Stizolobium aterrimum na presença de trifloxysulfuron -sodium & $\hat{\mathrm{Y}}=18,00$ & $=$ \\
\hline Stizolobium aterrimum na ausência de trifloxysul furon-sodium & $\hat{\mathrm{Y}}=18,01$ & - \\
\hline Canavalia ensiformis na presença de trifloxysulfuron-sodium & $\dot{\mathrm{Y}}=18,04$ & - \\
\hline Canavalia ensiformis na ausência de trifloxysul furon-sodium & $\hat{\mathrm{Y}}=18,05$ & - \\
\hline \multirow[t]{2}{*}{ Sem cultivo prévio e com presença de trifloxysulfuron-sodium } & $\hat{\mathrm{Y}}=2,5+2,11667 \mathrm{U}$ & 0,73 \\
\hline & Intoxicacão (\%) & \\
\hline Stizolobium aterrimum na presença de trifloxysulfuron -sodium & $\hat{\mathrm{Y}}=0,00$ & - \\
\hline Stizolobium aterrimum na ausência de trifloxysulfuron-sodium & $\dot{\mathrm{Y}}=0,00$ & - \\
\hline Canavalia ensiformis na presença de trifloxysulfuron-sodium & $\hat{\mathrm{Y}}=0,00$ & - \\
\hline Canavalia ensiformis na ausência de trifloxysul furon-sodium & $\hat{\mathrm{Y}}=0,00$ & - \\
\hline Sem cultivo prévio e com presença de trifloxysulfuron-sodium & $\hat{\mathrm{Y}}=87,53$ & - \\
\hline
\end{tabular}




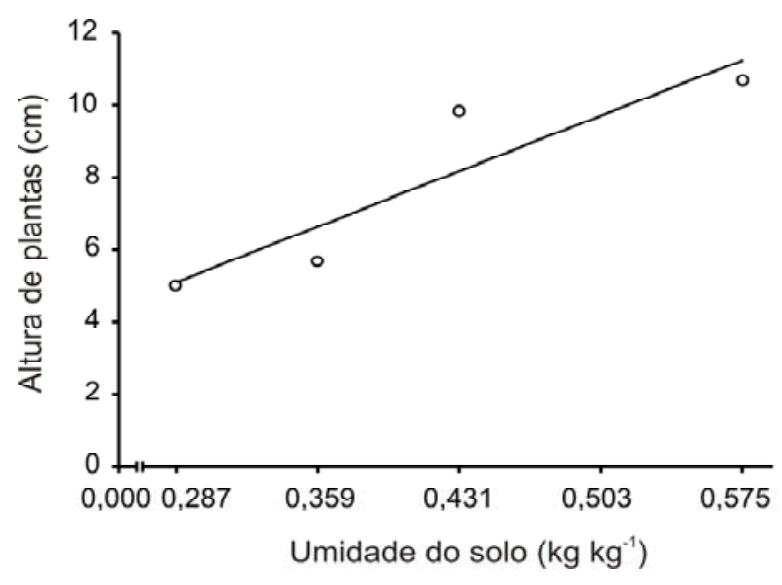

Figura 7 - Valor estimado e observado para altura de plantas de sorgo cultivadas em solo com aplicação de trifloxysulfuron-sodium, em função de diferentes níveis de umidade $(\mathrm{U})$, após 60 dias sem cultivo prévio de espécies fitorremediadoras $\left(\mathrm{o}-\hat{\mathrm{Y}}=2,5+2,11667^{*} \mathrm{U}\right.$, $\left.r^{2}=0,73\right)$.

\section{AGRADECIMENTOS}

Ao Conselho Nacional de Desenvolvimento Científico e Tecnológico - CNPq, pelo apoio financeiro para realização deste trabalho.

\section{LITERATURA CITADA}

BLANCO, H. G. Destino, comportamento e resíduos dos herbicidas no solo. Biológico, v. 45, p. 225-248, 1979.

\section{EMPRESA BRASILEIRA DE PESQUISA}

AGROPECUÁRIA - EMBRAPA. Manual de métodos de análise de solo. 2. ed. Rio de Janeiro: Centro Nacional de Pesquisa de Solos, 1997. 212 p.
FLURY, M. Experimental evidence of transport of pesticides through field soils: a review. J. Environ. Quality, v. 25, n. 1, p. 25-45, 1996.

LEVANON, D. et al. Mobility of agrochemicals through soil from two tillage systems. J. Environ. Quality, v. 22, n. 1, p. 155-161, 1993.

MUELLER, T. C.; SHAW, D. R.; WITT, W. W. Relative dissipation of acetochlor, alachlor, metolachlor, and SAN 582 from three surface soils. Weed Sci., v. 13, n. 1, p. 341-346, 1999.

OLIVEIRA, M. F. Comportamento de herbicidas no ambiente. In: OLIVEIRA Jr., R. S.; CONSTANTIN, J. Plantas daninhas e seu manejo. Guaíba: Agropecuária, 2001. p. 315-362.

PROCÓPIO, S. O. et al. Potencial de espécies vegetais para a remediação do herbicida trifloxysulfuron-sodium. Planta Daninha, v. 23, n. 1, p. 9-16, 2005.

ROCHA, W. S. D. Sorção de 2,4-D e diuron nos agregados organominerais de latossolos em função dos conteúdos de matéria orgânica e de água. 2003. 88 f. Tese (Doutorado em Solos e Nutrição e Plantas) - Escola Superior de Agricultura “Luiz de Queiroz", Universidade de São Paulo, Piracicaba, 2003.

RODRIGUES, B. N.; ALMEIDA, F. S. Guia de herbicidas. 5. ed. Londrina: Edição dos Autores, 2005. 591 p.

WALKER, A.; MOON, Y.; WELCH, S. J. Influence of temperature, soil moisture and soil characteristics on the persistence of alachlor. Pest. Sci, v. 35, n. 1, p. 109-116, 1992.

WEED, D. A. J. et al. Dissipation and distribution of herbicides in the soil profile. J. Environ. Quality, v. 24, n. 1, p. 68-79, 1995. 